\section{UTILITY OF DOBUTAMINE STRESS ECHO IN PREDICTING CARDIAC EVENTS; A RETROSPECTIVE AUDIT OF DSE'S AT THE MATER INFIRMORUM HOSPITAL, BELFAST}

${ }^{1}$ Patrick Savage, ${ }^{1}$ Michael Shahmohammadi, ${ }^{2}$ Suzanne Maynard, ${ }^{2}$ Brian McClements, ${ }^{3}$ Susane Mulholland. ${ }^{1}$ Cardiology Registrar, Mater Hospital, Belfast Trust, UK; ${ }^{2}$ Consultant Cardiologist, Mater Hospital, Belfast Trust, UK; ${ }^{3}$ Lead Cardiac Physiologist, Mater Hospital, Belfast Trust, UK

\subsection{6/heartjnl-2021-BCS.49}

Current NICE guidance recommends CT coronary angiography (CTCA) as first line for investigation of new onset stable chest pain, however; CTCA is not yet readily available in all UK centres. DSE remains recommended in current ESC guidance and provides a readily available, low cost alternative (1). Indeed, a negative test has been demonstrated to have an excellent negative predictive value in the region of $>98 \%$ (2). Aim We sought to evaluate the rate of cardiac events within two years of a negative DSE.

Methods We performed a retrospective data interrogation of all DSE's performed in the Mater Hospital Cardiac Investigations Department between 2017 and 2019. MACE was evaluated at two years. Data were extracted using local electronic healthcare records. Statistical analysis performed using SPSS software.

Results 302 DSE's were performed during the study period. The mean age was $64.1 \pm 10.4$ years with $41.7 \%$ male. Of the population, $16.2 \%$ had a prior history of IHD with $19.2 \%$ being diabetic. All tests were requested by the Cardiology team on an outpatient. 15 patients had a positive test. At two years the negative predictive value of a negative DSE was $98.3 \%$. A positive test had a sensitivity for predicting coronary artery disease of $86.7 \%$ with a false positive rate of approximately of $13.3 \%$. The overall complication rate was low at $0.7 \%$. Using a combined endpoint of time to ACS or revascularisation; there was a significant difference $(\mathrm{p}<0.001)$ in event free survival between groups (figure 1).

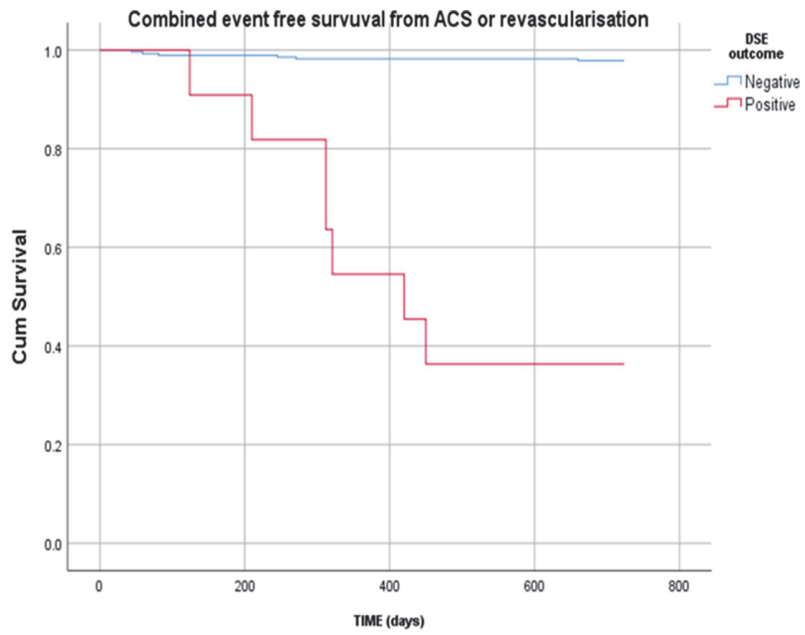

Abstract 49 Figure 1 Kaplan-Meier survival curve demonstrating freedom from combined endpoint of ACS or revascularisation. In patients with a negative test, mean event free survival was $719.3 \pm 9.3$ days. In those with a positive test, mean event free survival was 460.8 \pm 128 .9days. There was a significant difference in event free survival between groups $(p<0.001)$. Data expressed as mean $\pm 95 \% \mathrm{Cl}$. Variance between groups assessed using log rank Mantel Cox regression analysis
Conclusion DSE is a safe test with a high sensitivity for detecting coronary artery disease. Furthermore, compared to a positive test, a negative test has a strong negative predictive value for cardiac events at two years.

Conflict of Interest none

\section{TROPONIN IS INDEPENDENTLY ASSOCIATED WITH INCREASED SHORT-TERM MORTALITY IN HOSPITALISED PATIENTS WITH COVID-19 INFECTION: A RETROSPECTIVE STUDY IN AN INNER CITY LONDON HOSPITAL}

Vijay Shyam-Sundar, Dan Stein, Martina Spazzapan, Andrew Sullivan, Cathy Qin, Victor Voon. Homerton University Hospital Foundation Trust, London, UK

\subsection{6/heartjnl-2021-BCS.50}

Introduction Increased mortality is thought to be associated with an elevated troponin in addition to co-morbidities and age. International studies have demonstrated that troponin is an independent predictor of mortality in COVID-19 patients but to our knowledge this has not been assessed in a UK hospitalised population. We performed a single-centre retrospective observational study investigating the association between troponin positivity in patients hospitalised with COVID-19 and increased mortality in the short term.

Methods All adults admitted with swab-proven RT-PCR COVID-19 to Homerton University Hospital (HUH) from 04.02.20 to 30.04.20 were eligible for inclusion. We retrospectively analysed data collected from the physical and electronic patient records (EPR) including demographic and biochemical data (e.g. serum high sensitivity Troponin I). Data was analysed according to the primary outcome of death at 28 days during hospital admission. Troponin positivity was defined above the upper limit of normal according to our local laboratory assay $(>15.5 \mathrm{ng} / \mathrm{l}$ for females, $>34 \mathrm{ng} / \mathrm{l}$ for males). Univariate and multivariate logistical regression analyses were performed to evaluate the link between troponin positivity and death.

Results The total number of adults with swab-proven RT-PCR COVID-19 to HUH from the date of the first positive swab to 30th April 2020 was 402. Mean length of stay for all patients was 9.1 days(SD 12.0). Table 1 shows selected demographics. This is a highly comorbid population with modest ethnic minority representation. Mean age was 65.3 years for men compared to 63.8 years for women. In those with a positive initial troponin, there was a high burden of mortality at 28 days post-admission. Mortality in troponin positive and negative patients is shown in table 2. A chi-squared test

Abstract 50 Table 1 Selected demographics

\begin{tabular}{lll}
\hline & & Proportion(\%) \\
\hline Gender & Male & 53 \\
& Female & 47 \\
Ethnicity & Black/Asian & 36 \\
Co-morbidities & Hypertension & 54 \\
& Dyslipidaemia & 26 \\
& Diabetes mellitus & 37 \\
& Lung disease & 24 \\
& Chronic kidney disease & 18 \\
\hline
\end{tabular}

